\title{
Effekte des Authentischen? Selbstentwurf und Referenz in der Autobiographie Johanns von Soest (1504/05)
}

\begin{abstract}
Using the example of Johannes von Soest's rhymed autobiography of this contribution examines the historical text type of the "early-modern autobiography" and its specific pragmatic and narratological conditions. The theory proposed by PHILIPE LEJEUNE for modern autobiography of a trio of identities - author, narrator and protagonist - is rephrased and extended with regard to the early modern period. In addition, as far as text production is concerned, two aspects are central: first, on the level of written production, the close connection, which can be reconstructed from the manuscript, between the initial idea and its writing down in one's own hand; second, on the level of the narrative, the tension between the model for the narration and events in the author's life.
\end{abstract}

Die im zweiten Weltkrieg in Frankfurt verbrannte Handschrift Nr. 166 des Fichardschen Familienarchivs enthielt eine offenbar vom Verfasser selbst aufgeschriebene, retrospektive Lebenserzählung. Es handelt sich um die bekannte, Ende 1504 oder 1505 verfaßte Lebenserzählung des früheren Heidelberger Sängermeisters und späteren Frankfurter Stadtarztes Johann von Soest. ${ }^{1}$ Sie gilt als die erste nicht rollenmäßig stilisierte deutschsprachige Autobiographie. ${ }^{2}$. Gleichzeitig aber wurde ihre Abhängigkeit von konventionellen Erzählmustern betont. ${ }^{3}$ Der Text - das wird zu zeigen sein - stützt beide Auffassun-

ARTHUR WIEGAND, Die Handschrift Nr. 166 des Freiherl, von Fichardschen Familienarchivs als Grundlage für die Beurteilung von Sprache, Stil und Metrik des Johann von Soest. Diss. masch., Marburg 1922, S. 10-45; die erste Edition von Johanns von Soest Lebensbeschreibung wurde im Frankfurtischen Archiv für altere deutsche Literatur und Geschichte, hg. von JOHANN CHRISTIAN VON FICHARD. Bd. 1, Frankfurt a. M. 1811, S. 84-139 veröffentlicht [ein Wiederabdruck wurde von FRIEDRICH WILHELM AUGUST POTT im Jahrbuch des Vereins für Orts- und Heimatskunde in der Graffschaft Mark 17 (1902/03), S. 95-135 besorgt].

21. URSULA SCHULZE, Autobiographie (Deutsche Literatur), Lexikon des Mittelalters, Bd. 1, Sp. 1265-1268, hier 1267.

GESA BONATH, Johann von Soest, ${ }^{2}$ VL, Bd. 4, Sp. 744-755, hier Sp. 753-755; HoRsT BRUNNER, Johann von Soest, Willibald Pirckheimer - zwei Falsstudien, In Autorentypen, hg, von WALTER HAUG/BURGHaRT WACHINGER (Fortuna Vitrea 6), Tübingèn 1991, S. 89-103, hier S. 94. 
gen und regt dadurch dazu an, über die >Authentizitätı des Beschriebeneñn nachzudenken. Diese Überlegungen sind, wiewohl sie aus literaturwissen schaftlicher Sicht formuliert werden, als Reaktion auf eine überdisziplinäret Forschungslage anzusehen. Die vor allem von geschichtswissenschaftlicher Seite geförderte Erschließung der frühneuzeitlichen Autobiographie als menta litätsgeschichtlicher und mikrohistorischer >Quelle,${ }^{4}$ die " - verglichen min anderen Quellengattungen - einen direkteren Zugang zum historischen Indiw duum « ermögliche, ${ }^{5}$ trifft und verbindet sich mit literaturhistorischen Interes sen am historischen Texttyp und seinen speziellen pragmatischen und narratot logischen Bedingungen ${ }^{6}$ und mit der in der systematischen Autobiographiedis kussion zentralen texttheoretischen Frage nach der Referenz der Texte. Beidev Bereiche hängen eng mit dem Authentizitätsproblem zusammen. ${ }^{7}$

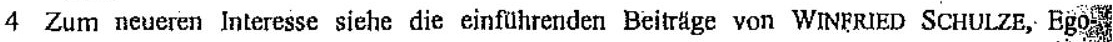
Dokumente: Annäherung an den Menschen in der Geschichte? Vorüberlegungen fụr die Tagung "EGO-DOKUMENTE«, in: Ego-Dokumente. Annäherungen an den Menschen in dets Geschichte, hg. von WINFRIED SCHULZE (Selbstzeugnisse der Neuzeit 2), Berlin 1996, S. 11 30; BENIGNA VON KRUSENSTJERN, Was sind Selbstzeugnisse? Begriffskritische und quellent kundliche Überlegungen anhand von Beispielen aus dem 17. Jahrhundert, Historische Anthrou pologie 2 (1994), S. 462-471; SABINE SCHMOLINSKX, Selbstzeugnisse im Mittelalter, in: Das dargestellte Ich. Studien zu Selbstzeugnissen des späteren Mittelalters und der frühén Neuzuitu hg. von KLAUS ARNOLD/SABINE SCHMOLINSKY/URS MARTIN ZAHND (Selbstzeugnisse ded Mittelalters und der beginnenden Neuzeit 1), Bochum 1999, S. 19-28; FABIAN BRÄNDLE [ux a.], Texte zwischen Erfahrung und Diskurs. Probleme der Selbstzeugnisforschung, in: Von det dargestellten Person zum erinnerten Ich. Europäische Selbstzeugnisse als historische Quellen (1500-1850), hg. von KASPAR VON GREYERZ [u. a.] (Selbstzeugnisse der Neuzeit 9). Köln I[u a.] 2001, S. 3-31.

5 KASPAR VON GREYERZ, Deutschschweizerische Selbstzeugnisse (1500-1800) als Quellen de Mentalitätsgeschichte. Bericht über ein Forschungsprojekt, in: ARNOLD 1999 [Anm. 4], S. 147] 163, hier S. 151. Es geht ihm um Sozialisation, Konfession, Krankheitserfahrungen und kollek tive Einstellung zum Tod (S. 151). BRÄNDLE [u. a.] 2001 [Anm. 4], S. 12, sind der Meinung gi daß »Selbstzeugnisse als schriftlicher Ausdruck von Selbstbetrachtung einen Zugang $z_{i m}$ historischen Menschen mit vergleichsweise hoher Authentizitłt« bieten, räumen aber die Intêt ferenz »der kulturellen oder gațungsmäßigen Stilisierung« ein.

6 HANS RUDOLF VELTEN, Das selbst geschriebene Leben. Eine Studie zur deutschen Autobiö graphie im 16. Jahrhundert (Frankfurter Beitrăge zur Gertnanistik 29), Heidelberg 1995, ist'dfe ergiebigste germanistische Monographie zu diesem Thema. STEPHAN PASTENACI, Erzăhlfoimin und Persönlichkeitsdarstellung in deutschsprachigen Autobiographien des 16. Jahrhundertst Ein Beitrag zur Historischen Psychologie (Literatur, Imagination, Realitat 6), Trier 1993, bietêt viele anregende Beobachtungen; diese Arbeit ist aber in den theoretischen Voraussetzungen problematisch, da der Versuch, eine historische Psychologie zu rekonstruieren, an der unpșy chologischen، Darstellungsweise der meisten frílhneuzeitlichen Autobiographien vorbeigeht; (s etwa die problematische psychologisierende Deutung zu Thomas Platters Autobiographie, is 186-189).

7 Daß die Konstruiertheits des Textes, seine Stilisierung und seine gattungsmäßige Vorausset zung für eine als, Quelle wahrgenommene Autobiographie bei der Analyse bedacht werden müssen, ist auch in geschichtswissenschafticher Wahrnehmung akzeptierte Voraussetzung vgl. etwa SCHULZE 1996 [Anm. 4], S. 24f., und die bindige Zusammenfassung bei EcKHART. HENNING, Selbstzeugnisse, in: Die archivalischen Quellen: Eine Einführung in ihre Benutzing gh hg. von FRIEDRICH BECK/ECKHART HENNING (Veroffentlichungen des Brandenburgischeng Landeshauptarchivs 29), Weimar ${ }^{2} 1994$, S. 107-114, bes. S. 107f,; vgl. allgemeiner und nicht 
Die Begriffe, die meine Untersuchung leiten, sind Verschriftlichungsprogramm und Effekt des Authentischen. Unter Verschriftlichungsprogramm Werstehe ich eine oder mehrere wirksame Darstellungsstrategien, welche sichthaŕludie retrospektive Präsentation eines erinnerten Lebens im schriftlichen Text leiten. Dieses Programm kann - dies ist hier der Fall - aus einem narrati7. Schema abgeleitet sein. Effekt des Authentischen bezeichnet einen textuelfen Effekt, ${ }^{8}$ der den Einđruck eines unverstellten Blickes auf das faktische Weben der schreibenden Person, auf deren Erinnerung der eigenen Vergangenheit, auf deren Welt- und Selbstsicht oder auf deren Welt- und SelbstdeuImingsmuster entstehen läßt. Der Eindruck von Unmittelbarkeit wird durch bestimmte textuelle Verfahren erzeugt, ist abhängig von Inszenierungen, ist halso nicht wirklich unmittelbar, sondern weist vielmehr sehr deutlich auf Strategien; auf Mittelbarkeit, auf Herstellung hin. Verschiedene Aspekte spielen bet dieser Herstellung eine Rolle: die Referenz (Jauthentischer Werke dokumentieren historische Fakten), der Gestaltungsfaktor (>Authentizität kann durch Gestaltungsmittel erzeugt werden) und die Frage der Qualifizierung des Urithebers (etwa Augenzeugenschaft), also der Zusammenhang von Autorität (Mnd Authentizität. ${ }^{9}$ Es muß darum gehen, innerhalb des Textes Differenzierungenivorzunehmen, anhand derer ein textuelles Relief sichtbar wird, in dem auf Jeweils unterschiedlichen Ebenen Effekte des Authentischen wirksam sind. Es ist freilich evident, daß die Wirkung dieser Effekte ganz auf die pragmatische Dimension des Textes bezogen ist. Der Effekt ist - auch wenn er, wie ich zeigén will, an intrinsischen Textmerkmalen sichtbar/wird - abhängig von der Situation. von Konventionen und von (intendierten)

\section{II.}

Ein wichtiger pragmatischer Rahmen frühneuzeitlicher Autobiographien ist die familiäre Hausüberlieferung. Als solche »an das Haus gebundene Literatur " ${ }^{10}$ Whernehmen die retrospektiven Lebenserzählungen oft erklärtermaßen didakti-

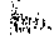

speziell auf autobiographisches Schriftum bezogen HANS-WERNER GOETZ, Moderne Mediävistik. Stand und Perspektiven der Mittelalterforschung, Darmstadt 1999, S. 169 f.

Helmut LeTHEN, Versionen des Authentischen: sechs Gemeinplatze, in: Literatur und Kul䈍: turwissenschaft. Positionen, Theorien, Modelle, hg. von HaRTMUT BöHME/KLAUS R. M. SCHERPE, Reinbek bei Hamburg 1996, S. 205-231, hier S. 209.

粼 Eine Typologie des >Authentischen hat MATIAS MARTINEZ, Authentizităt als Kunstlichkeit in at Steven Spielbergs Film Schindler's List, Compass - Mainzer Hefte für Allgemeine und VerWgleichende Literaturwissenschaft 2 (1997), S. 36-60, hier S. 36-42, vorgelegt. .Von den ediittionsphilologischen, theologischen und juristischerr Begriffsbestimmungen von Authentizität Wgrenzt er einen ästhetischen Authentizitätsbegriff ab, der sich auf eine $»$ Eigenschaft von if Kunstwerken « (S. 38) beziehe, und unterscheidet für den asthetischen Bereich die drei »Bedeuwit tungsaspekte«, die ich hier aufgreife.

O!GRRHARD WOLF, Autopoiesis und Autorpoesie, Zur Funktion des Autors in fruhneuzeitlichen HiHauschroniken, in: Fragen nach dem Autor. Positionen und Perspektiven, hg. von FELIX PHILIPP INGOLD/WERNER WUNDERLICH. Konstanz 1992. S. 61-71. hier S. 62. 
sche Funktion: Sie sollen häufig als Exempel für die nachfolgende Generation dienen. ${ }^{11}$ Wenngleich dies im Text selbst nicht ausgesprochen wird, wart Johanns von Soest Autobiographie sicherlich als Lebenshilfe für die kom mende Generation gedacht. ${ }^{12} \mathrm{Zu}$ dieser Funktion tritt der Wunsch nach bleit bender Erinnerung über den Tod hinaus. ${ }^{13}$ Die didaktische Funktion läßt sich aber kaum bis in die erzählten Einzelheiten verfolgen. Vielmehr scheint es das geschriebene Leben als Ganzes zu sein, fuir das exemplarische Geltung bean sprucht wird. Exemplarisch ist nicht unbedingt das. Selbstverständnis des Ver fassers: "viel mehr als die Suche nach dem Selbstverständnis war für die Ver/4 fasser die Mitteilung und Darstellung ihres Lebens und ihres Handelns von Bedeutung. ${ }^{14}$ Die Teilhabe an Ereignissen ist Gegenstand der Darstellung Würde man also als genuines Merkmal der Autobiographie ansehen, daß sich der Exzähler Rechenschaft über sinnere〈 Vorgänge oder gar eine sinnere wicklung ablege (dies würde die Gattung historisch stark begrenzen), müßté man wohl eintäumen, daß es dann die frühneuzeitliche deutschsprachige Auto biographie kaum geben könne. Trotzdem läßt sich festhalten, daß die Autobiö graphie Johanns von Soest auch Elemente eines geschlossenen Lebensent wurfes und Ansätze einer Selbstreflexion bietet. Der Selbstentwurf; den diese frühneuzeitliche Autobiographie als Textform repräsentiert, ist jedoch nicht in der Weise gebündelt, daß die persönliche Identität als Einheit sichtbar wird Gleichzeitig ist sie aber auch kein bloßes Konglomerat von auf das dargestellte Ich bezogenen Ereignissen. ${ }^{15}$ Für Johanns Lebenserzählung, wie wohl allget mein für die frühneuzeitliche Autobiographie, wird man eine Art textuelles Kontinuum annehmen müssen, in welchem beides - das Ereignis-Konglomera

$11 \mathrm{Vgl}$ allgemein JUDITH POLLMANN, Religious Choice in the Dutch Republic. The Reformation of Amoldus Buchelius (1565-1641). Manchester/New. York 1999, S. 20; VELTEN 1995 [Anm] 6], S. 89-91 (zu Konrad Pellikan); zum exempelhaften Charakter der Lebenserzăhlung Thomás Platters vgl. ebd., S. 105; Sigmund von Herberstein etwa spricht davon, daß seine autobiograj phischen Schriften "Exempeln geben sollen, in deren fueststapfen die Nachkommen tretent müßten« (HARALD TERSCH, Österreichische Selbstzeugnisse des Spätmittelalters und def Frühen Neuzeit (1400-1650). Eine Darstellung in Einzelbeiträgen, Wien [u, a.] 1998, S. 196f( die Zitate finden sich in Johannes Tichtels Tagebuch, Sigmunds von Herberstein Selbstbiogra. phie, Johannes Cuspinians Tagebuch, Georg Kirchmairs Denkwürdigkeiten, hg. von THEODOR GEORG VON KARAJAN [Fontes rerum Austriacarum 1,1], Wien 1855, S. XIV).

12 BRUNNER 1991 [Anm. 3], S. 94.

13 Fol. XV', 39 (F 114). Die Zuverlässigkeit der Edition WIEGANDs [Anm. 1] bezweifelt MEI NOLF SCHUMACHER, Ein , Geistliches Jahr, um 1500. Die Sonn- und Festtagsgedichte des Johann von Soest, ZfdA 122 (1993), S. 425-452, hier S. 432 Anm. 49, wahrscheinlich zurecht Die maschinengeschriebene Dissertation (UB Marburg, Sign. XVI B Wiegand, Arthur) weist zudem viele (wohl von WIEGAND selbst?) eingetragene handschriftliche Verbesserungen in Editionsteil auf: Auch wenn WIEGANDs Edition aufgrund ihres wissenschaftlichen Anspruches, sicher zuverlässiger ist, bleibt ein Vergleich mit FICHARDs Ausgabe [Anm. 1] ratsam (hier Sigle F mit Seitenzahl); WIEGANDs Apparat der Abweichungen gegenilber FICHARDS Ausgabe ist - wie Stichproben ergeben - nicht zu trauen.

14 VELTEN 1995 [Anm. 6], S. 3.

$15 \mathrm{Vgl}$. zur Systematik dieser Unterscheidung ALOIS HAHN, Identität und Selbstthematisierung in: Selbstthematisierung und Selbstzeugnis. Bekenntnis und Geständnis, hg. von ALois HAHN/VOLKER KAPP. Frankfurt a. M. 1987, S. 9-24, hier S. 16. 
wie die oftmals eher angedeutete als durchgeführte Konstruktion einer autobiographischen Einheit - in je unterschiedlicher Intensität sichtbar wird. yt Johanns von Soest Autobiographie ist - etwa im Gegensatz zur autobiographischen Form des Diarium - eine Autobiographie im eigentlichen Sinn, ${ }^{16}$ nămlich retrospektive Erzählung oder Rekonstruktion dessen, was dem Verfasser und sicher auch den zunächst wohl auf den weiteren Familienkreis beschränkten Rezipienten ganz unverdächtig das erinnerte Leben des sich selbst Ich nennenden Verfassers ist. Ich bin weit davon entfernt, mich in die uferlose Diskussion um eine Gattungsdefinition der frühneuzeitlichen Autobiographie einzumischen, ${ }^{17}$ will aber dennoch eine ungefäbre Angabe machen, was ich mit Autobiographie im. vorliegenden Zusammenhang meine. Eine retrospektive Erzählung verfolgt die Lebensgeschichte des Protagonisten von Geburt oder Kindheit bis zum Zeitpunkt der Niederschrift; diese mrückblickende Schreib-situation « ist ein entscheidendes Merkmal in der narrativen Organisation, denn sie bildet "einen markierten Standort, von welchem aus der Autor sein Leben narrativ organisiert und interpretiert. ${ }^{18}$ Ein weiteres grundlegendes Kititerium, dies hat vor allem VELTEN herausgearbeitet, ist der pragmatische Aspekt der Autobiographie. Er ist entscheidend für die Beurteilung der Frage, ob und wann eine Autobiographie als solche wahrgenommen wird; dies gilt sowohl für die historische Rezeption als auch für die heutige Wahrnehmung Wh:

翟 6 Zwei gute neuere Überblicke zur Geschichte und Theorie der Autobiographie findet man in fiden Einfuihrungen von MICHAELA HOLDENRIED, Autobiograqhie (RUB 17624, Literaturstudium), Stuttgart 2000, und MARTINA WAGNER-EGELHAAF, 㹡itobiographie (sI 323), Stutt-

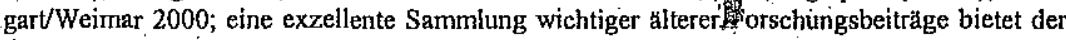
Band: Die Autobiographie. Zu Form und Geschichte einer literarischen Gattung, hg. von GÜNTER NigGL (WdF 565), Damstadt 1989.

W7: Es gibt einen großen Unschärfebereich im Kontakt mit anderen, ebenso nur vage bestimmbaren Gattungen wie dem Reisebericht oder den Hausbüchern (in denen sich häufig auch die mehr oder weniger in sich geschlossenen Autobiograptien finden). Es geht mir hier vor allem darum, anhand der Kriterien die Grundbedingungen für die Interpretation des Textes zu sichem, und weniger um eine Gattungsklassifizierung, die aufgrund der fehlenden Gattungsgeschlossenheit und der vielen transgenerischen Formen in diesem Bereich zum Scheitem verurteilt wäre. Vgl. z. B. zur Mischung von-Reisebericht und Autobiographie die Überlegungen HANS-JOCHEN SCHIEWERs anhand Hans Schiltbergers Hafterzählung (Leben unter Heiden. Hans Schiltbergers türkische und tartarische Erfahrungen, Daphnis 21 (1992), S. 159-178, hier S. $171 \mathrm{f}$.). Die Klassifizierung von festen Subgenres bei denjenigen frithneuzeitlichen Schriften, die sich im weiteren Sínne dem Autobiographischen zuordnen lassen, scheint unmöglich: $n$ It has proved impossible to classify early modem autobiographical texts into fixed genres 1. (POLLMANN 1999 [Anm. 11], S. 17); vgl. auch VELTEN 1995 [Anm. 6], S. 11-13.

19.8 VELTEN 1995 [Anm. 6], S. 21; vgl. auch S. 84f. Dieser folgt hier JÜRGEN LEHMANN, Bekennen 称 1988, S. 37, und vor allem den grundlegenden Beobachtungen DILTHEYs (Die Selbstbiographie, in: Ders., Der Aufbai der geschichtlichen Welt in den Geisteswissenschaften, hg. von BERNHARD GROETHUYSEN (WILHELM DILTHEYs gesammelte Schriften 7), Leipzig/Berlin 1927, S.199-202, hier S. 201f.). Für VELTEN manifestiert sich in dieser vom Text entworfenen Schreibsituation der Autor als organisierendes und interpretierendes Prinzip; ich meine, auf dieser (textuellen) Ebene kann man gesichert nur vom Erzähler sprechen. Mit diesem Problem allerdings gerät man in die mise en abîme-Struktur der Autobiographie, die aus der konstruierten Identität von Autor, Erzähler und Hauptperson entsteht; vgl. auch unten Anm. 20. 
der Zeugnis- oder Quellenfähigkeit der Gattung. Die Autobiographie kon struiert eine Identität des bezeichneten Protagonisten mit dem Erzähler; zusät lich konstruiert sie eine Identität des 'Autors mit dem Erzähler und dem Prota' gonisten. ${ }^{19}$ Erst diese textuelle Konstruktion erzeugt die Annahme, in ihrer Lebenserzählung erzähle die auch in anderen Schriftstücken historisch belegte Person Johann von Soest über ihr (tatsächlich gelebtes) Leben. ${ }^{20} \mathrm{Zu}$ dieser Identitätstrias tritt der mediale Aspekt, daß frühneuzeitliche Autobiographien häufig als Autographe überliefert sind oder überliefert waren. Dies war auch für Johanns Text der Fall. ${ }^{21}$ In der Identitätskonstruktion bildet sich - auf einer anderen Ebene - die mediale Situation in der Instanz des Schreibers ab. Es handelt sich auch in diesem Sinne um ein $»$ selbst geschriebenes Leben ${ }^{22}{ }^{22} \mathrm{Da} \beta$ in dieser Konstellation die Hand des Schreibers sowohl zum Körper einer historischen Person gehört, als auch an der Schrift der Lebenserzählung diesèr Person mitwirkt, ist die entscheidende (trennende und verbindende) >Schwelles zwischen Text und Mensch: Die Vorstellung eines )Authentischen( entsteht nicht zuletzt aus dieser Verbindung.

Auch wenn PHILIPPE LeJEUNE die mediale Dimension aufgrund seiner Ausrichtung auf die neuere Autobiographie nicht im Blick hatte, läßt sich seine These, daß sich das Funktionieren dieser Konstellation pragmatisch, ïbèr Konventionen entscheidet, auf die frühneuzeitliche Autobiographie übertra gen. ${ }^{23}$ LEJEUNE spricht in diesem Zusammenhang von einem autobiographischen Pakt. Auf die Situation der fruihneuzeitlichen Autobiographie ange, wendet: Eine historisch belegte Person läßt sich namentlich mit dem Erzähler identifizieren, der behauptet, nun sein eigenes Leben zu erzählen; zusätzlich kann unter Umständen die Schreiberhand als diejenige derselben historischen Person identifiziert werden. Zunächst aber wird deutlich, daß es bei heutigen Interpreten eines Bewußtseins darüber bedarf, daß auch sie einen autobiogra phischen Pakt eingehen, sobald sie den Text als referentiellen wahrnehmen dies tun sie immer, wenn sie ihn als >Zeugnis〈, als sDokument " oder äls ২Quelle sehen. Wird oder bleibt ihnen dieser pragmatische Pakt unsichtbar dann laufen sie Gefahr, das erzäblte Leben als Zeugnis oder Dokument zu deuten; das einen unmittelbar sauthentischen Eindruck der Welt- und Selbst

19 PHILIPPE LEJEUNE, Der autobiographische Pakt, in: Der autobiographische Pakt, hg. von P LEJEUNE (edition suhrkamp N. F. 896), Frankfurt a. M. 1994, S. 13-51, hier S. 14.

$20 \mathrm{Zu}$ betonen bleibt, daß es sich hierbei um die $\mathrm{K}$ o n $\mathrm{s} t \mathrm{r}$ u $\mathrm{k}$ t i o n der Identität handelt. Vom erzähltheoretischen Standpunkt her können die Instanzen erzähltes Ich, erzahlendes Ich und shistorischec Person (realers Autor) nicht eins sein.

21 Dies kann WIEGAND I922 [Arm. 1], S. 97-110, aufgrund einer detaillierten Untersuchung plausibel machen.

22 Ich bezjehe mich auf den Titel der Monographie VELTENs 1995 [Anm. 6]. Die Genese de frühneuzeitlichen Autobiographie wird - sicher zurecht - mit der gesteigerten Lese- und Schreibfähigkeit und der Verfügbarkeit von.Schreibmaterialien in Verbindung gebracht; vg] SCHULzE 1996 [Anm. 4], S. 19; RUCHARD VAN DULLMEN, Die Entdeckung des Individuring 1500-1800, Frankfurt a. M. 1997, S. 12, 15f: Johann von Soest war mit der Produktion von Schriftum im weiten Sinne gut vertraut (vgl. BRUNNER 1991 [Anm. 3]).

23 LEJEUNE 1994 [Anm. 19]. 
wahrnehmung des Verfassers oder der Verfasserin vermitteln kann. Bleibt ihnen der Rahmen, der autobiographische Pakt, deutlich, dann sehen sie die Autobiographie als das, was sie ist: Sie konstruiert den Eindruck, ein authentisches Zeugnis der Welt- und Selbstwahrnehmung eines historischenc Menschen zu sein. Wo heutige Interpreten glauben, das >Zeugnis extrapolieren zu können, sehen sie doch nur die dieses Zeugnis konstruierende Struktur in ihrer unhintergehbaren Textualität. ${ }^{24}$ Identifizieren sie das İch des Textes mit einer historischen Person und leiten-daraus eine /Wahrnehmungs der Welt oder zumindest ein Wahrnehmungsmuster $a b$, bewegen sie sich bereits innerhalb der Konstruktion. Hier liegt ein entscheidendes methodisches Problem.

\section{III.}

Über das Leben Johanns unterrichtet vor allem seine Lebensbeschreibung, zudem sind Dokumente überliefert, die mit seiner Tätigkeit als Sängermeister in Heidelberg und als Arzt zusammenhärigen. ${ }^{25}$ In allen Lebensstadien - spätestens seit dem Aufenthalt in Kassel - betätigt sich Johann offenbar auch als Dichter: Es sind eine Reihe weiterer. Werke Johanns exhalten. ${ }^{26}$ Über diese Tätigkeit wird in den erhaltenen Teilen der Autobiographie aber nichts gesagt. Ich gebe einen knappen, ergänzungsbedürftigen Eindruck des Lebenslaufes: ${ }^{27}$ Johann yon Soest wird in Unna geboren, früh schon fällt seine stimmliche Begabung auf, die ihm den Weg an den Herzoghof in Kleve ebnet. Er erhält weitere Stimmausbildung und Anstellungen in Utrecht, Hardenbergh und Maastricht, hält sich später in Kassel und als Sängermeister am pfalzgräflichen Hof in Heidelberg auf. Über die nächste Phase seines Lebens ist wenig bekannt, wohl hat Johann eine medizinische Ausbildung genossen. Schließlich

24 Dies ist fiur die Autobiographie vor allem im Zuge der Rezeption poststrukturalistischer Theoreme (und deren kritischer Sichtung) ausgeführt worden; vgl. ALMUT-FINCK, Autobiographisches Schreiben nach dem. Ende der Autobiographie (Geschlechterdifferenz und Literatur 9), Berlin 1999, S. 23-76. Ich will damit - um es deutlich zu sagen - nicht in Abrede stellen, daß sich innerhalb des Textes Spuren von sozialen Praktiken finden. So etwa korreliert - um nur ein Beispiel unter vielen zu nennen - in der unten, S. 276, besprochenen ersten erzählten Begebenheit in der Autobiographie Johanns die Wallfaht der Mutter mit den zahlreichen historischen Belege für Frömmigkeitspraxis und Wunderglauben. Diese Spuren aber finden sich innerhalb der beschriebenen Konstruktion und sind zunächst innerhalb dieser zu beurteilen.

25 Belege im Zusammenhang mit Johanns Sängertatigkeit am pfalzgraflichen Hof bei GERHARD PLETZSCH, Quellen und Forschungen zur Geschichte der Musik am kurpfalzischen Hof zu Heidelberg bis 1622, in: Akademie der Wissenschaften und der Literatur in Mainz, Abh. der geistes- und sozialwiss. KI. 1963, 6, Mainz 1963, S. 583-763, hier S. 621, 650, 678-682 (dort auch weitere Informationen żur Familie). Zur Tätigkeit in Frankfurt WIEGAND 1922 [Anm. 1], S, 107-109, mit älterer Literatur, und HORST WENZEL, Die Autobiographie des späten Mittelalters und der frühen Neuzeit. Bd. 2: Die Selbstdeutung des Stadtbürgertums (Spätmittelalterliche Texte 4), Múnchen 1980, S. 82.

26 Ein.Profil von Johann von Soest als Autor erstellt BRLNNER 1991 [Anm. 3].

27. Zur Ergänzung siehe WENZEL [Anm. 25], S. 81-84; BONATH [Anm. 3], Sp. 744-746; BRUNNER 1991 [Anm. 3], S. 91-95. 
wird er Stadtarzt in Worms, später in Oppenheim, dann im Jahre $1500^{28}$ in in Frankfurt, wo er im Dezember 1504 oder im Jahr 1505 seine Lebensretrospek tive aufschreibt. Extrahiert man allerdings - wie ich es zum Teil getan habe aus der Lebensbeschreibung die Daten in dieser Weise, läuft man Gefahr, dié Literarizität des Textes zu unterschlagen. Den retrospektiven Lebensbeschrei: bungen liegt immer auch Erzählstrategie zugrunde, >Gemachtheits ist ein Charakteristikum dieser Texte wie jedes erzäblenden Textes - dies ist umso. deutlicher zu betonen, als die frühneuzeitliche Autobiographie zwar in den Zusammenhängen einer pragmatischen Schriftlichkeit zu sehen ist, aber sich in starkem Maße solcher Schemata bedient, die im weiteren Sinne als >literarischi zu bezeichnen sind. ${ }^{29}$ Bevor diese Gemachtheit ins Zentrum der Aufmerksamkeit rücken kann, muß aber zunächst der pragmatische Kontext, der Status der Handschrift und ihr Umfeld erläutert werden.

Die verlorene Handschrift, in der Johanns Autobiographie überliefert war; zeigte, wie WIEGAND an vielen Beispielen deutlich machen kann, in all ihrei Teilen Merkmale einer Konzepthandschrift, die den Arbeitsprozeß des Ver fassers widerspiegelt. ${ }^{30}$. Sie enthielt nicht nur die Autobiographie: Auch überliefert waren ein Lobgedicht auf die Stadt Frankfurt, Gedichte über die Sonn: und Feiertagsperikopen ${ }^{31}$ und eine Apothekertaxe. Diese Texte verweisen in unterschiedlichè pragmatische Zusammenhänge. Die Apothekertaxe (also eine Art Gebührenordnung) bezieht sich wohl auf Johanns Tätigkeit als Arzt. Eś handelt sich um eine Abschrift der offenbar im Jahr 1500 (fol. LXXVII ${ }^{ }$), dem Jahr der Bestallung Johanns, vom Rat der Stadt Frankfurt erlassenen Apothekertaxe. Die Gedichte auf die Perikopen scheinen, so MEINOLF SCHUMACHER in seinem Beitrag, Ausdruck einer Frömmigkeitspraxis zu sein. ${ }^{32}$ Das Loblied auf die Stadt Frankfurt hängt wohl mit der Bestallung Johanns durch den Răt zusammen. $^{33}$. Ein Teil von unbekannter Größe der Handschrift war schon ver

28 Die Ennenung ist urkundlich festgehalten; vgl. WIEGAND 1922 [Anm. 1], S. $107 \mathrm{f}$.

$29 \mathrm{Vgl}$. HANS-JÜRGEN BACHORSKI, Der selektive Blick. Zur Reflexion von Liebe und Ehe in Autobiographien des Spätmittelalters, in: Eheglïck und Liebesjoch. Bilder von Liebe, Ehe und Familie in der Literatur des 15. und 16. Jahrhunderts, hg. von MARIA E. MULLER (Ergebnisse der Frauenforschung 14), Weinheim/Basel 1988, S. 23-46, bes. S. 38-39.

30 Es ist der Dissertation von WIEGAND 1922 [Anm. 1] zu verdanken (auf die WENZEL 1980 [Anm. 25], S. 86, und SCHUMACHER 1993 [Anm. 13] hingewiesen haben), daß wir uns ein ver gleichsweise genaues Bild der Handschrift machen können, in der Johanns von Soest Lebenș erzählung enthalten war. Zur Charakterisierung der Handschrift vgl. WIEGAND, S. 4 und 97 110 (von "Koncept《 spricht WIEGAND auf S. 97; vgl. auch SCHUMACHER, S. 449): »Folioblät: ter aus Papier sind in der Mitte gefaltet und zu ganz ungleichen Lagen vereinigt. Die Blattgross se ist also etwa $11 \times 33 \mathrm{~cm}$. Dabei sind die Blätter nicht einmal alle gleich gross. Einige sind unten abgerissen. Das Abreissen der Stücke ist aber nicht etwa später geschehen, sondern bevor die Seiten beschrieben wurden. Ferner sind kleinere Zettel in die Handschrift eingelegt, auf denen der Verfasser Verbesserungen und Nachträge zu dem Texte niedergelegt hat:« (WiE) GAND'S. 97). WIEGAND venmutet, daß die Verse während des Dichtens selbst geschrieben wurt den.

$31 \mathrm{Vgl.zu}$ diesen SCHUMACHER 1993 [Anm. 13].

32 Ebd., S. 450. 
loren als FICHARD die Lebensbeschreibung 1811 zum ersten Mal edierte; aufgrund dieses Verlustes ist ein Teil der Lebensbeschreibung nicht exhalten. Über eine wie immer geartete Rezeption der Handschrift ist nichts bekannt. Sie verblieb sicherlich ganz im familiären Bereich. In diesem Kontext ist auch die Autobiographie zu sehen.

Daß Johann Schreiber und Verfasser der Handschrift war, kann WIEGAND ${ }^{34}$ wahrscheinlich machen. In der Autobiographie findet sich eine Namensnennung (fol. $X^{r}, 23-30 ;$ F 98) zusammen mit der Erklärung des Rufnamens Johann von Soest bzw. sústchen und eine Selbstbenennung des Sprechers in einem erimerten Dialog (fol. XXII', 6f.; F 126); die Autobiographie ist mit $j$. signiert (fol. XXVI $) ;{ }^{35}$ dieses Kürzel findet sich in der Handschrift an einer weiteren Stelle, um die Schreiber- und Autorschaft Johanns zu bezeichnen. ${ }^{36}$ An vielen anderen Stellen der Handschrift wird das Sprecher-Ich (bzw. Schreiber- bzw. Verfasser-Ich) mit dem Namen Johann von Soest bezeichnet. ${ }^{37}$ Eine Identifizienung der historischen Person des Frankfurter Stadtarztes mit dem Protagonisten und dem Erzähler der Autobiographie ist WIEGAND aufgrund der Apothekertaxe möglich. Diese in der Handschrift mitïberlieferte Abschrift, sdie in ihren deutschen Teilen die Hand Johanns von Soest verrät ${ }^{38}$ und in der dieser auch namentlich erwähnt ist, bezieht sich auf historisch nachweisbare ${ }^{39}$ Tätigkeiten und Interessen des Arżtes Johann von Soest. ${ }^{40}$ Die Möglichkeit eines autobiographischen Paktes, der aus der Konstruktion eines Identitätsquartetts (in der Namensidentität Protagonist - Exzäbler - Schreiber - statsächliche( Person) entstehen kann, ist also für Handschrift und Text nachvollziehbar. Die Autorität des Geschriebenen ergibt sich aus einer Kombination won Autographie und Zeugenschaft, aus der Tatsache, daß eine selbst geschriebene, signierte Schrift entsteht, deren Inhalt auf der Erinnerung des selbst erlebten Lebens basiert.

Gestützt wird diese Autorität durch die Applikation eines exempelhaften oder legendarischen Musters als grundlegendes Verschriftlichungsprogramm. Es ist die Anlehnung an das geistliche Muster, die, so scheint es, das Sprechen

34. WIEGAND 1922 [Anm. 1], S. 97-110.

135 LEJEUNE 1994 [Änm. 19], S. 19 und 23, identifiziert die »Signaturs (in einem weiteren Sinne)

Ift als entscheidendes Mittel, um den autobiographischen Pakt zu etablieren.

36 WIEGAND 1922 [Anm. 1], S. 107 (compositum j. fol. XXXIV , 22): WIEGAND gibt das. Kürzel in seiner handschriftlichen Form wieder.

137 Belege zusammengestellt ebd., S. $106 \mathrm{f}$.

38 Ebd., S. 108.

解9 Vgl. ebd., S. $107 f$.

40 Aus dem Grabstein Johanns, den WIEGAND (ebd., S. 108f.) als weiteres Argument ins Feld ma führt, dagegen läßt sich eine solche Identifizierung des Arztes und Dichters nicht unbedingt abyit: leiten. In der Inschrift Hic ex susato Steivyert cubat ecce Johannes, / Cantanti et medica DocWy tor in arte potens bezieht sich in der Aussage, daß Johann ein Doktor war, welcher der Kunst Wi w des >Singens` und der Heilkunst mächtig war, die erste Zuschreibung, die u. U. auch mit i. isKunst des Dichtens « wiedergegeben werden könnte (so begreift sie WIEGAND), vielleicht eher 
über sich selbst zu legitimieren hilft. ${ }^{41}$ Ich werde zunächst diese Applikation beschreiben, um dann auf die Elemente des Textes einzugehen, die quer zum Verschriftlichungsprogramm der Autobiographie liegen. Die Strukturienung des Lebenslaufs in dieser Autobiographie ist sicherlich das auffälligste konzeptionelle Merkmal. Nach kurzem Bericht über Herkunft und Benuf des Và̆ ters und über die Familie geht die Beschreibung zu dem über, was der Erzähler als myn erste abentwr (fol: VI ${ }^{v}, 7 ; \mathrm{F}$ 86) bezeichnet: Das kleyne kynt (fol: VI 6; F ebd.) Johann zieht sich, so wird erzählt, aufgrund eines Unfalls mit hei ßem Öl eine schwere Gesichtsverletzung zu und wird blind. Die Mutter macht mit dem Kind eine walfart (fol. $\mathrm{VI}^{v}, 13 ; \mathrm{F}$ ebd.) und bringt ein Opfer, um zu erreichen, daß es nicht dauerhaft erblinde. Darauf bessert sich sein Zustand, und das rechte Auge erholt sich wieder, während die linke Gesichtshälfte ver sehrt und das entsprechende Auge blind bleibt (fol. $V^{v}, 17-24 ; \mathrm{F}$ ebd.). Auch wenn die Kausalität nicht explizit gemacht wird, kann kein Zweifel bestehen; daß zwischen Wallfahrt und Heilung des rechten Auges eine direkte Bezie: hung geknüpft wird. So bietet dieses erste erzählenswerte Lebensereignis einen zeittypischen Beleg für den Glauben an die heilsame Wirkung von Wallfahit und Opfer. ${ }^{42}$ Bedeutsam in erzählstrategischer Hinsicht ist die Stellung der Episode als die erste abentwr der Lebenserzählung. Sie gibt ein Grundthema vor: Die providentielle Aufgehobenheit des Ich, die durch die Initialstellung in der ersten abentwr besonders herausgehoben präsentiert wird.

Die verlorene Handschrift machte - wie WIEGAND gut dokumentiert - durch die eingefügten kleineren Zettel, durch die an den Rand geschriebenen Zusätzê und durch die zahlreichen Streichungen und Verbesserungen die Niederschrift als zeitlichen Prozeß sichtbar. Textproduktion ist hier beides: Niederschrift und Dichten in zeitlich engst möglicher Verschlingung. Es gibt Spuren, idaß Johann von Soest im ersten Teil an der Stimmigkeit des narrativen Gesanit entwurfs gearbeitet hat. Bemerkenswert ist in diesem Zusammenhang eine nicht direkt in der ersten. Niederschrift enthaltene, offenbar hinzugefügte Passage. In der Handschrift war zu fol. VII (dem zweiten Blatt der Lebensbe-

41 Über die Legitimation eines mittelalterlichen self-fashioning des Einzelnen auf der Grundlage eines Lebens, das exemplarischen Charakter hat, weil es dem exemplarischen Muster (indiesem Fall Christi) folgt, vgl. JEFFrey F. HAMBURGER, Medieval Self-fashioning: Authorship Authority, and Autobiography in Suso's Exemplar, in: Ders, The Visual and the Visionaryi Ant and Female Spirituality in Late Medieval Germany, New York 1998, S. 233-278, bes. S 2766 278.

$42 \mathrm{Zu}$ Unfall und Krankheit als Motivation von Wallfahrten in der Fruhen Neuzeit ${ }^{2}$ sieheb BARBARA SCHUH, >Alltags und >Besonderheite spatmittelalterlicher und fruhneuzeitlichener Wunderberichte, in: Wallfahrt und Alltag in Mittelalter und Früher Neuzeit. Internationales Round-Table-Gespräch, Krems an der Donau, 8. Oktober 1990, hg. von GERHARD JARIT/ BARBARA SCHUH (Sitzungsberichte der österr. Akademie der Wissenschaften, phil.-hist Klas $^{4}$ se, 592), Wien 1992, S. 255-276, hier S. 263-267. Die Heilungsverheißung dominiert auch die für die Wallfahrtsorte swerbende und Wallfahrt, ebd., S. 95-113, hier S. 96-99. Vgl, auch REBEKKA HABRRMAS, Wallfahrt und Aufruhr. Zur Geschichte des Wunderglaubens in der frühen Neuzeit (Historische Studien 5 ) Frankfurt a. M./New York 199i, S. 49-54, zu Wallfahrten mit Kindem, die einen Unfall hatten 
schreibung) ein $» B e i b l a t t$ von der Grösse $15 \times 6 \mathrm{~cm} \ll,{ }^{43}$ also etwa halb so hoch und halb so breit wie der überwiegende Teil der Handschrift, mit Versen in der Hand Johanns eingeheftet. Geschildert wird in diesem Zusatz, wie der neunjährige Protagonist sich - nach anfänglicher guter Entwicklung als Chorsänger - willentlich von einem Gaukler entführen läßt, der verspricht; ihn sein Handwerk zu lehren, und wie er von Soester Soldaten, die ihn suchen, wieder zurückgebracht wird. Deutlich sichtbar wird das Auseinandertreten von erinnertern Ich und erzählendem Ich in der retrospektiven distanzierten, leicht kritischen Beurteilung der Motivation für die Begleitung des Gauklers:

Das dy tzijt wasz myn grosz begyr.

Syn konst, dy hett ich gern gelert.

Mich ducht, ich wer da durch geert.

(fol. VII', 50-52; F 89)

Diese Episode ist die erste Abweichung - sie ist als solche auf der Handlungsebene räumlich umgesetzt - von einem als >richtig» stilisierten Weg. Deutlich angelegt ist das Motiv des jugendlichen Intums, das später zu dem bestimmenden Motiv des ersten Teils ausgebaut wird.

In der Folge sind die dann sehr viel deutlicheren Abwertungen der ersten Jahre offenbar direkt in die Niederschrift eingegangen. Positiv gewertet wird das Verbleiben in festen Ordnungsrahmen (etwa, als der Protagonist im Dienst des Klever Herzogs das Fundament seiner Karriere als Sänger und Komponist legt); negativ gewertet werden die direkt auf diesen Erfolg zurückgeführten Ausweise von hoffart und bosheyt (fol: $\mathrm{X}^{\mathrm{r}}, 1-22$; F 97). Der erste Teil der Autobiographie erweist sich so als stimmig: Nach der Evokation des providentiellen Musters in der Heilung des rechten Auges folgt später die jugendliche Ver-irrung. Einem legendarischen Muster entsprechend, wird so erzählt, daß die Wende des Lebens, an "der sich der Sprectier von den >Jugendsünden< lossagt, motiviert erscheint.

Diese Konstruktion erweist sich als Teil der gut sichtbaren erzählerischen Grundstruktur der Lebensbeschreibung. HORST BRUNNER hat sie so zusammengefaßt: Die Autobiographie stilisiert das Leben des Helden mnach dem Schema: wilde Jugend - Umkehr - Gottergebenheit ${ }^{44}{ }^{4}$ Diese Grundstruktur GESA BONATH bezeichnet sie als geistliches Schema ${ }^{45}$ - bestimmt das ErzähIen von Anfang an. Der Text ist um sie herum organisiert wie um einen sinnstiftenden Kern und verdankt ihm seine zweiteilige Struktur. Ein im Rhythmus des Ambrosianischen Hymnus gehaltenes lateinisches Gebet, das programmaצtisch mit den Worten Delicta iuventutis mee / Ne memineris domine beginat and in dem der Sprecher wegen seiner Jugendsünden Abbitte leistet, ist die 
Scheide. ${ }^{46}$ Defuzitär ist das, was diesem Gebet vorausgeht. Dagegen ist dass ander buch, das nach diesem Gebet und der entsprechenden moralischen Be-1 lehrung folgt, von Anflügen göttlicher Vorsehung geprägt. Es sind dort keinèrtu lei Momente einer Selbstkritik vorhanden. ${ }^{47}$ Auf diese Anlage baut eine ganzż dominante Appellstruktur des Textes. Die Funktion des Reims, eines auffälli: gen und fuir Lebensbeschreibungen unüblichen Sprachmittels, ${ }^{48}$ ist wohl im Zusammenhang mit der legendenhaften Moralisierung des Lebenswegs $z \mathrm{it}$ sehen: Die gebundene Sprache verweist auf Traditionen der lehrhaften Rede ${ }^{\text {th }}$

Die Applikation des legendarischen Musters auf eine säkulare Biographie ist nun keineswegs außergewöhnlich für die frübneuzeitliche Autobiographię Das exzählende Ich gestaltet die Konversion als die bewußte Wendung, welche mit einer Uberzeugung korreliert, daß das Frühere falsch oder defizient, das Spätere (und Jetzige) richtig sei. Dies ist weit verbreitet im religiösen Schriffil tum, ist repräsentativ für spirituelle Erneuenung. ${ }^{49}$ Aber auch Erzählungen einer wie immer akzentuierten Veränderung im Weltdasein des Menschen (also losgelöst von im engeren Sinne religiösen Erfahrungen) können nach diesem Modell gestaltet sein: "Conversion narratives as a way of explaining personal change are deeply embedded in Western culture ${ }^{50}{ }^{50}$ Das erzählende Ich bezeichnet die ersten vierundzwanzig Jahre des exinnerten Lebens als bóslich verthon (fol. $\mathrm{XV}^{\mathrm{v}}, 3 ; \mathrm{F}$.114). Wendepunkt ist Johanns Bestallung als Sängermeister in Heidelberg und seine erste Ehe, auf die hier vorausgewiesen wird. ${ }^{51}$ In einem metanarrativen Kommentar wird die zweiteilige Strukturt

46 Fol. $X V^{v}, 9-42$; F 114f. Dazu kommt noch eine moralisierende Jugendbelehrung auf deutsch (fol. XVI'-XVII'; F-115-119).

$47 \mathrm{Vgl}$. auch BONATH [Anm. 3], Sp. 754.

48 Die frühneuzeitlichen autobiographischen Texte sind nicht so gut erschlossen, daß man einerlo Überblick über deutschsprachige gereimte Autobiographien bekommen könnte; TERSCH 1998 [Anm. 11] verzeichnet für den österreichischen Bereich (1400-1650) nur die gereimte Auto biographie des Krainer Landeshauptmanns Joseph von Lamberg (S. 214-225); zu erwähnen wäre noch (neben den autobiographischen Liedern Oswalds von Wolkenstein und Michei] Beheims) die gereinte Autobiographie Georg Nieges (Leben im 16. Jahrhundert. Lebenslauf und Lieder des. Hauptmanns Georg Niege, hg. und kommentiert von BRAGE BEI DER WIEDEN [Selbstzeugnisse der Neuzeit 4], Berlin. 1996), auch wenn es weitere Beispiele geben muß, ist der Anteil gereimter autobiographischer Schriften sicherlich vergleichsweise gering.

49 Diese ,frommer Selbststilisierung Johanns, so die begründete Vèrmutung SCHUMACHERs 19.93 [Anm. 13], S. 450-452, könnte in Verbindung mit der Frömmigkeitspraxis stehen, die aus den in derselben Handschrift überlieferten und wohl auch in der Abfassungszeit nahestehendert Perikopengedichten Johanns hervorscheint.

50 JUdith POLLMANN, A Different Road to God: The Protestant Experience of Conversion in the Sixteenth Century, in: Conversion to Modemities: the Globalization of Christianity, hg von PETER VAN DER VEER, London/New York 1996, S. 47-64, hier S. 47. Entscheidend ist der ret th rospektive, nachträglich ordnende Gestus im Erzählen; dies läßt sich schon für die frúhen christlichen Konversionserzähilungen sagen; in diesem Zusammenhang hat PAULA FREDRIKSEN (Paul and Augustine: Conversion Narratives, Orthodox Traditions and the Retrospective Self Journal of Theological Studies 37 (1986), S. 3-34) den Begriff des retrospective self( gepragt.

51 BONATH [Anm. 3], Sp. 754, stellt zurecht fest: wDa 21 Jahre seiner Biographié fehlen, ist nicht auszumachen, ob er seine Wandlung als plötzliche conversio oder als allmähliches Reiferwer

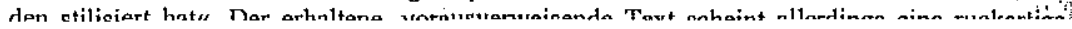


explizit bezeichnet. Auffällig ist, daß an diesem Scheidepunkt der Text mit der etwas unvermittelten direkten Anrede an ein unbestimmtes Du eine Art Dialogsituation entwirft:

\section{[...] eyn junckfraw ich da nam,} Durch welche ich da glich wart tzam. In welcher mosz das selb geschag, Hab ich gelegt auch in den tag Im andern buch nach dyssem teyl, Da yn das leèsz von tzyl zu tzyl, So vyndestu vil gutter sweng. Da selbest du myn by bedenck. (fol. XV $\mathrm{XV}^{\mathrm{r}}, 32-39 ; \mathrm{F} 113 \mathrm{f}$.)

Dort, im anderen Teil (dem zweiten Buch) des Lebens, so der Kommentar, ist das Gute zu finden. Der Appell an das unbestimmte Du ist, Johann nach diesem Buch seines Lebens zu beurteilen, sich seiner anhand dieses Lebensabschnittes - und nicht des ersten - zu erinnern. In den direkt folgenden Versen wird der erste Teil nun explizit beendet:
Also hostu das leben myn
Von anbegyn gedichtet fyn
Byss zu der tzijt, mych eben merck,
Das ich mich fogt gen Heydelberg.
(ebd., 40-43; F 114)

Ist im ersten buch des Lebens also ein kohärenter Entwurf eines insgesamt vertanen Lebens gelungen, sind im Abschnitt, der vom zweiten buch erhalten ist, dagegen Friktionen zu beobachten: Die Stimmigkeit ist nicht mehr ohne weiteres gegeben. Das Verhältnis von exemplarischen Mustern und Abweichungen im Beschriebenen wird hier besonders spannungsreich. Da das zweite buch nur fragmentarisch überliefert war, müssen Aussagen über die Stilisierung des gesamten Textes immer unter Vorbehalt stehen.

$\therefore$ Beschrieben wird in diesem zweiten Teil - oder in dem, was von ihri erhalten war (fol. XX'-XXVI'; F 119-139) - die Trauer um die gestorbene erste Frau, der Plan, Geistlicher zu werden und dessen Scheitem, Brautwerbung und Heirat der zweiten Frau, dann, unverhältnismäßig breit, die Streitigkeiten mit deren Vater und Querelen bei Hofe, die zum Weggang Johanns aus Heidelberg führen. Zwar charakterisiert das erzählende Ich das erinnerte Leben nach der Wende als eines, das frei ist von den Irrtümern und Vergehen der Jugend, ja die Wende und der Wunsch, Geistlicher zu werden (fol. XX, 13; F 120), passen sich sehr gut ins legendarische Schema ein. Allerdings mischt sich in diese Stilisierung im zweiten Teil eine andere Tendenz im Erzählten. Die dort in: :

11. Veränderung im Sinne einer conversio nahezulegen (eyn junckfraw ich da nam, / Durch welche 
extensiv geschilderten Streitigkeiten sind nicht unbedingt dazu angetan, dem von Selbstkritik freien Sprecher die vom Muster vorgezeichnete Läuterung wirklich abzunehmen.

In diesen Passagen wird nämlich ein neuer Charakterzug des erzählten Ich ausgestellt. Es geht darum, Recht zu haben und Recht zu behalten. Die Let bensbeschreibung erzählt folgendes: Zunächst sichert sich Johann durch eine Art illegaler Hausbesetzung das seiner jungen Frau als Mitgift versprochene Haus (fol. XXIV, $30-42 ;$ F 133f). Im Streit mit seinem Schwiegervater - an diesem Streit nimmt, so der Text, ganz Heidelberg Anteil - müssen der Erzähi lung zufolge mehrere Räte der Stadt vermitteln, bis der Streit schließlich vo: Gericht geregelt wird (fol. XXIV ${ }^{v}-X^{\prime} V^{r} ;$ F 133-135). Auch in der nächsten Passage geht es um Selbstbehauptung: Als Johann, so wird erzählt, vom már, schalck (fol. $\mathrm{XXV}^{\mathrm{T}}, 17$; F 136) des pfalzgräflichèn Hofes öffentlich bei Tisch verhöhnt wird, vom Pfalzgraf (Philipp dem Aufrichtigen) Klärung der Angele genheit fordert, und von diesem die Antwort erhält, myn lyber knecht, / Lasz dich nyt yrren solche sach (ebd., 24f.; F ebd.), bleibt er beleidigt und unver söhnlich, bittet um orlob (ebd., 29; F ebd.), der ihm nach einem Jahr gewährt wird, und verläßt Heidelberg für immer. Insgesamt entsteht das Bild eines unversöhnlichen, auf seinem Recht stark beharrenden Menschen, der auch illegale oder illegitime Aktion ergreift, wenn es um dieses Recht geht. Dieses Bild paßt gar nicht zur Stilisierung des geläuterten Menschen Johann, wie es das Verschriftlichungsprogramm vorgibt. ${ }^{52}$ Ich betone noch einmal, daß ich hier nichts herausgreife. Diese Szenen sind neben der Brautwerbung ${ }^{53}$ die prominenten Partien des zweiten Teils. Selbst wenn wit nicht wissen, wie die erste Passage dieses zweiten Teils ausgesehen hat, so läßt sich doch von den erhaltenen Passagen her das Bild des Geläuterten nicht einfach mit dem Erzählten in Einklang bringen.

Zwar exklärt der dem Gegenstand der Erzählung entsprechend stark involvierte Erzähler die Ereignisse der Jugend für defizitär und sieht dèn zweiten

52 Man könnte allerdings, blickt man etwa auf die 'Fehd und Abenteuer Götz' von Berlichingeñ, meinen, auch dieses Querulantentum sei etablierte Stilisierung in Selbstentwurf, die Abweichungen seien also ebenfalls in einem Schematismus aufgehoben. Die Autobiographie Johanns stützt aufgrund, ihrer völlig anderen thetorischen Struktur diese Meinung indes nicht: Götz; wapologetische Autobiographie« (UlRICH MÜLLER, Thesen zu einer Geschichte der Autobio: graphie im deutschen Mittelalter, in: Autobiographie [Anm, 16], S. 297-320, hier S. 307) dient dazu, ein fehdereiches Leben zu rechtfertigen und die Rechtmäßigkeit der Fehden zu begrin: den; Johanns Aútobiographie ist ein ganz anderer Fall, da die Erzahlung der Auseinandersetzungen nicht gerechtfertigt wird. Zu erwähnen bleibt, daß, wie POLLMANN 1999.[Anm. 11]; S. 17f., an verschiedenen frühneuzeitlichen Autobiographien zeigen kann, sogar krasses Fehlverhalten des dargestellten Ich manchmal ohne jeden relativierenden Kommentar erzählt wird.

53 Diese läßt sich als einzige Passage einigermaßen ins Verschriftlichungsprogramm einordnen; da die Brautwerbung durch eine Traumvorausdeutung als providentiell erscheint (BONATH [Anm. 3], Sp. 754). Vgl. die Analyse dieser Passage bei BACHORSKI 1988 [Anm. 29], S. 36f, dieser sieht in Werbungsgespräch sliteratisch stilisierte[n] Verkehrsfomen einer kulturellen und sozialen Elite anzitiert; das ist, blickt man auf die Passage, vielleicht etwas hoch gegriffen; allerdings stilisiert sich Johann zum vollendeten Brautwerber, die Szene paßt also gut in das ander buch, in den sgelungenen< Lebensteil. 
Teil des Lebens als gefestigt und geläutert an. Gerade aber die späteren Ereignisse in der Erzählung stemmen sich gegen diese Deklaration. Diese Ereignisse - das ist in der Diskussion um textuelle Effekte des Authentischen nicht unwichtig - stehen in Zusammenhang mit der Thematisiening starker Emotionen. Hängt das Durchkreuzen des Verschriftlichungsprogramms durch Elemente, die sich diesem nicht fügen, mit dieser Thematisierung zusammen? Diese Frage ist methodisch kontrolliert wohl nicht za beantworten; das Verhältnis von frühneuzeitlicher Autobiographik und der Repräsentation yon Emotionen ist ein gerade erst entdecktes Forschungsfeld. ${ }^{54}$ Der Text stelit jedenfalls (implizit) die Eignung des exemplarischen Schemas für die Darstellung des Lebens Johanns in Frage. Dies ist, meine ich, entscheidend: Das eigene Leben wird zum Thema und nach einem legendarischen Programm verschriftlicht. $\mathrm{Daß}$ sich die Lebensanlage dafür nicht eignet, erzeugt - selbst dann, wenn man eine historisch von der heutigen verschiedene Kohärenzerwartung ansetzt Spannung: Unter dem legendarisch-exemplarische Muster dringt eine methodisch äußerst schwer zu fassende Eigentümlichkeit des Erzählens hervor. $\mathrm{Zu}$ beobachten ist ein Widerstreit zwischen dem offensichtlichen narrativen Kalkuil und dem Erzählten, das dieses Kalkül unterminiert. JAN-DIRK MüLLER hat für das frühneuzeitliche autobiographische Exzählen allgemein festgehalten: »Ưm ibre Erfahrungen in die sinnvolle Ordnung dẹ Narration zu bringen, verwenden die autobiographischen Exzähler bestimmte Verlaufsschemata und Deutungsmuster. Diese sind weniger prägnant und konsequent als im Roman nachweisbar, weil sie von anderen Darstellungsabsichten - der Dokumentation und Präsentation kontingenter Lebensumstände - durchkreuzt werden. ${ }^{55}$ Ich meine, man kann beobachten, daß in Johanns von Soest Autobiographie der kohärente Lebensentwurf eher im legendarischen Muster, also in der Stilisierung des Lebens, als in der Präsentation kontingenter Lebensumstände entsteht. Wenn aber im zweiten Buch dem Muster entgegenstehende kontingente Ereignisse erzählt werden, entsteht ein Effekt, den man als Effekt des Authentischen bezeichnen könnte. Die Spannung zwischen Verschriftlichungsprogramm und diesen ihm entgegenstehenden Erzählelementẹn bleibt unaufgelöst. ${ }^{56}$ Der Effekt entsteht >gegen< das Verschriftlichungsprogramm, wird vielleicht nicht einmal shergestellt‘, sondern ist ein Überschußphänomen. Es mag sein, daß es im 16. Jahrhundert die Konstruktion von Authentizität, ja ihre

54 Vgl. BRÄNDLE [u. a.] 2001 [Anm. 4], S. 11f. (2Emotion als Selbstzeugnis - Selbstzeugnis als Emotion(); die Autoren weisen auf die methodischen Unsicherheiten in der Erforschung dieses zit: Bereiches hin.

55 JAN-DIRK MÜLLER, Der Prosaroman - eine Verfallsgeschichte? Zu Clemens Lugowskis Ana.lyse des $>$ Formalen Mythos\& (mit einem Vorspruch), in: Mittelalter und fruhe Neuzeit. Übergänge, Umbralche und Neuansätze, hg. von WALTER HaUG (Fortuna vitrea 16), Tübingen 1999, S. 143-163, hier S: 154.

56. Es ist zu beachten, daß Elemente von autobiographischen Texten munterdeterminiert» sein t.. können: »nicht jedes Detail [ist] in einen funktionalen Zusammenhang eingebunden« (ebd. S. 152); die Spannung ließe sich auch beschreiben als eine Spannung zwischen der Determination a: des Lebenslaufs im legendarischen Muster und der Unterdetermination der abweichenden Ele-

if: mente innerhalb dieses vom Muster gestifteten Zusammenhangs. 
intendierte literarische Herstellung. (etwa im Prosaroman) gibt. ${ }^{57}$ In dieser Lebensbeschreibung allerdings ist sie ein nicht vom Verfasser intendierter; hergestellter Effekt. Agens dieses Effekts ist eine jenseits des Verschriftlichungsprogramms liegende gegenläufige Bewegung des Textes. Hier liegt das Problem im häufig benutzten Begriff der >Textstrategies: Der an diesem Text beobachtete Effekt des Authentischen folgt keiner Strategie, vielmehr stellt er sich g e g e n die offensichtliche Darstellungsstrategie ein. Daher wäre auch der Begriff der Authentizitäts s $t \mathrm{r}$ a $\mathrm{t}$ e $\mathrm{g}$ i e hier verfehlt.

Doch ist die Vorstellung; daß gerade die Abweichung vom Muster in dieser Autobiographie einen Effekt des Authentischen auslöse, historisch angemessen? $\mathrm{Zu}$ bedenken ist das, was CAROLINE BYNUM für Texte des 12. Jahrhunderts und JEFFREY HAMBURGER für Seuses Vita und deren Rezeption ermittelt haben. ${ }^{58} \mathrm{Zu}$ beobachten ist an diesen mittelalterlichen Texten (und Bildwerken), daß diese gerade einen an Mustern oder Modellen ausgerichteten Selbstentwurf des Einzelnen anstreben, daß also das Profil des Einzelnen nicht in der Differenz, sondern in der Imitation eines institutionalisierten, überpersönlichen Modells entsteht. ${ }^{59}$ Darf man also nicht vor diesem historischen Hintergrund der exemplarischen Struktur der Lebenserzählung, der Konversionsstruktur, eher den Anspruch zubilligen, einen Effekt des Authentischen zu erzeugen? Die Lösung ist wohl, daß man dies für beide zentralen Merkmale des Textes für das Muster wie für die Abweichungen - annehmen muß. Der Identitäts: entwurf in diesem Text baut sich geradezu auf in der Spannung zwischen Verschriftlichungsprogramm und den sich nicht fügenden Elementen. Vielleicht ist der eigentliche Effekt des Authentischen zu sehen in dieser Spannung, die keinen einfachen Identitätsentwurf zuläßt: Weder wird nämlich wirkliche Individualität in Weltsicht und Selbstentwurf sichtbar (dies zu behaupten, würde bedeuten, die Dominanz des Exemplarischen zu vernachlässigen), noch läßt sich der Entwurf ganz auf die Imitation kollektiver Muster beschränken (dies verhindern die sich nicht fügenden Elemente). Vielleicht also verweist der Text gleichzeitig auf die musterfolgenden wie auf die differenzsetzenden Aspekte eines Identitätsentwurfes, vielleicht inszeniert er Identität einerseits als Reflex kollektiver Ordnung und andererseits als gegenläufige Spuren von Autonomie in einem in sich nicht kohärenten Aufbau. ${ }^{60}$

57 Vgl. ebd., S. 151-155, bes. S. 152.

58 CAROLINE WALKER BYNUM, Did the Twelfth. Century Discover the Individual?, Journal of Ecclesiastical History 31 (1980), S. 1-17; HAMBURGER 1998 [Anm. 41].

59 Der Text würde dann also auf eine Art von sMentalitätı referieren. In diesem Sinne zur frühneuzeitlichen Autobiographie BACHORSKI 1988 [Anm. 29], S. 39: wDie literarischen Strukturen und Topoi artikulieren in ihrer historischen Gewordenheit schon per se Partikel kollektiver Mentalität und die darin enthaltenen Ordnungs- und Interpretationsschemata; das jeweils sprechende Individuum läßt sich bei ihrer Verwendung jedoch nur soweit erkennen, wie es an dieser kollektiven Mentalität tejlhat.« Gleichzeitig bietet der Text - ganz ähnlich wie HAMBURGER 1998 [Anm. 41] dies furr Seuse beschreibt - durch seinen Anspruch, selbst exemplarisch zu sein, die Möglichkeit der Weitergabe des Modelis.

60 Zu dieser Spannung in der Wahmehmung des sSelbstseins in der Frühen Neuzeit vgl. VERENA

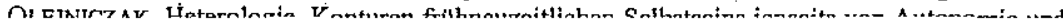


V.

Auf dem nun erreichten Stand der Analyse bleibt ein Fragezeichen stehen. Es betrifft die weiter oben aufgestellte These, über den >authentischen< Status eines Textelements oder eines Textes würde analog zum autobiographischen Pakt pragmatisch-konventionell entschieden. Hier ergibt sich ein methodisches Problem: Außer der grundlegenden Tatsache, daß die Konstruktion einer Identität von Protagonist, Erzähler, Schreiber und shistorischer، Person wohl auch im historischen Kontext authentizitätsstiftend gewirkt hat, können Vermutungen über einen Effekt des Authentischen in Johanns Autobiographie bloß aufgrund der Interpretation und Analyse des Textes selbst angestellt werden. Daß in der Spannung der unterschiedlichen Identitätsaspekte ein Effekt des Authentischen zu sehen ist (und dies ist die Bedingung dafür, Johanns Autobiographie als ıZeugnis ` dieser Spannung zu begreifen), läßt sich zwar aufgrund der sich in der Forschung abzeichnenden sKonturen frühneuzeitlichen Selbstseins ${ }^{61}$ plausibel machen, letztlich aber ruht die Annahme der $>$ Authentizitätı auf einen methodisch nicht zu sichernden >Pakt ‘ zwischen heutigem Interpreten und dem Text. Warum sollte man dann überhaupt von Effekten des Authentischen in der Autobiographie ausgehen und sich nicht doch mit der Textualität und mit der fehlenden Möglichkeit, Referenz und Authentizität methodisch gesichert zu erforschen, abfinden? Die Antwort liegt in der Besonderheit der Gattung begründet. Wir müssen die Frage nach dem Authentischen stellen, weil die Autobiographie, auch die der Frühen Neuzeit, wie kein anderer Texttyp mit dem Potential des Authentischen operiert und weil in dieser Gattung der (historisch in der deutschen Literatur zu diesem Zeitpunkt gerade entstehende) Fall auftritt, daß das oben beschriebene autobiographische Identitätsquartett konstruiert wird, welches ein Nachdenken über die Authentizität des gesamten Textes einfordert. Ich meine, darin liegt der.Grund, daß, wie MiCHAELA HOLDENRIED in ihrer Einführung bemerkt, "die Frage nach der >Authentizität` eines Textes $[. .$.$] weiterhin ein wichtiges Kriterium { }^{62}$ in der Autobiographieforschung geblieben ist. ${ }^{63}$

Heteronomie, LiLi 10.1 (1996), S. 6-36, besonders die exzellente forschungsgeschichtliche und systematische Analyse auf S. 6-12.

61 Ebd.

62 HOLdENRIED 2000 [Anm. 16], S. 26.

63 Nach Abschluß dieses Beitrags erschien eine für den vorliegenden Zusammenhang wichtige Publikation, die leider nicht mehr berücksichtigt werden konnte: Zeitenblicke I (2002), Nr. 2 [20.12.2002], URL: http://www.zeitenblicke.historicum.net/2002/02/index.html (12.03.2003). Diese hat die Erforschung frühneuzeitlicher Ego-Dokumente zum.'Thema. Die Bedeutung der Publikation liegt vor allem darin, daß sie das längst überfällige und für das Thema unverzichtbare interdisziplinäre Gespräch zwischen Geschichts- und Literaturwissenschaft anstößt. Sie versarnmelt Studien und Projektberichte zu autobiographischen Schriften der Frühen Neuzeit 